\title{
Nanodroplet-Mediated Histotripsy for Image-guided Targeted Ultrasound Cell Ablation
}

\author{
Eli Vlaisavljevich ${ }^{1}$, Yasemin Yuksel Durmaz ${ }^{1}$, Adam Maxwell ${ }^{2}$, Mohamed ElSayed ${ }^{1,3}$, Zhen Xu ${ }^{1,4}{ }^{\bowtie}$ \\ 1. Department of Biomedical Engineering, University of Michigan, Ann Arbor, MI, USA; \\ 2. Department of Urology, University of Washington School of Medicine, Seattle, WA, USA; \\ 3. Macromolecular Science and Engineering Program, University of Michigan, Ann Arbor, MI, USA; \\ 4. Department of Pediatrics and Communicable Diseases, Division of Pediatric Cardiology, University of Michigan, Ann Arbor, MI, USA.
}

$\triangle$ Corresponding author: Mohamed E.H. ElSayed, Ph.D. University of Michigan, Department of Biomedical Engineering, 1101 Beal Avenue, 2150 Lurie Biomedical Engineering Building, Ann Arbor, MI 48109, USA. Phone: + 1 (734) 615-9404 Fax: + 1 (734) 647-4834 E-mail: melsayed@umich.edu Web: www.bme.umich.edu/centlab.php OR Zhen Xu, Ph.D. University of Michigan, Department of Biomedical Engineering, 2107 Carl A. Gerstacker Building, 2200 Bonisteel Boulevard, Ann Arbor, MI 48109, USA. Phone: + 1 (734) 647-4961 Fax: + 1 (734) 939-1905 E-mail: zhenx@umich.edu Web: www.bme.umich.edu/labs/xulab.

( ) Ivyspring International Publisher. This is an open-access article distributed under the terms of the Creative Commons License (http://creativecommons.org/ licenses/by-nc-nd/3.0/). Reproduction is permitted for personal, noncommercial use, provided that the article is in whole, unmodified, and properly cited.

Received: 2013.05.18; Accepted: 2013.09.10; Published: 2013.10.20

\begin{abstract}
This paper is an initial work towards developing an image-guided, targeted ultrasound ablation technique by combining histotripsy with nanodroplets that can be selectively delivered to tumor cells. Using extremely short, high-pressure pulses, histotripsy generates a dense cloud of cavitating microbubbles that fractionates tissue. We hypothesize that synthetic nanodroplets that encapsulate a perfluoropentane (PFP) core will transition upon exposure to ultrasound pulses into gas microbubbles, which will rapidly expand and collapse resulting in disruption of cells similar to the histotripsy process but at a significantly lower acoustic pressure. The significantly reduced cavitation threshold will allow histotripsy to be selectively delivered to the tumor tissue and greatly enhance the treatment efficiency while sparing neighboring healthy tissue. To test our hypothesis, we prepared nanodroplets with an average diameter of $204 \pm 4.7 \mathrm{~nm}$ at $37^{\circ} \mathrm{C}$ by self-assembly of an amphiphilic triblock copolymer around a PFP core followed by cross-linkage of the polymer shell forming stable nanodroplets. The nanodroplets were embedded in agarose tissue phantoms containing a sheet of red blood cells (RBCs), which were exposed to 2-cycle pulses applied by a $500 \mathrm{kHz}$ focused transducer. Using a high speed camera to monitor microbubble generation, the peak negative pressure threshold needed to generate bubbles $>50 \mu \mathrm{m}$ in agarose phantoms containing nanodroplets was measured to be $10.8 \mathrm{MPa}$, which is significantly lower than the 28.8 $\mathrm{MPa}$ observed using ultrasound pulses alone. High speed images also showed cavitation microbubbles produced from the nanodroplets displayed expansion and collapse similar to histotripsy alone at higher pressures. Nanodroplet-mediated histotripsy created consistent, well-defined fractionation of the RBCs in agarose tissue phantoms at $10 \mathrm{~Hz}$ pulse repetition frequency similar to the lesions generated by histotripsy alone but at a significantly lower pressure. These results support our hypothesis and demonstrate the potential of using nanodroplet-mediated histotripsy for targeted cell ablation.
\end{abstract}

Key words: Histotripsy, nanodroplets, cavitation, therapeutic ultrasound, targeted cell ablation.

\section{Introduction}

Many tumor types exhibit characteristically permeable vasculature with average endothelial gaps typically between $200-600 \mathrm{~nm}$ and show poor lym- phatic drainage, which is collectively known as the enhanced permeation and retention (EPR) effect [1-4]. To take advantage of the EPR effect, phase change 
nanodroplets have been shown to extravasate across tumor's leaky vasculature and enter the interstitial space to directly target cancer cells [5-11]. Nanodroplets encapsulate a perfluorocarbon (PFC) core that is stabilized by albumin, lipid, or polymer shells [5-8]. The PFC core typically has a boiling point lower than $37^{\circ} \mathrm{C}$, but nanodroplets remain in the liquid form at body temperature [12-14]. After passing through the leaky microvasculature to a tumor's interstitial space, these nanodroplets can be vaporized by ultrasound to form gas bubbles through acoustic droplet vaporization (ADV) [12-14]. Attachment of optimal targeting ligands allows nanodroplets to specifically bind to cancer cells [12, 14, 15]. Research has shown the use of nanodroplets and ADV for targeted imaging [10, 14-17], drug delivery [10, 11, 14-17], and induce cavitation to enhance ultrasound thermal therapy [18-20].

One of the biggest limitations for current nanodroplet-mediated drug delivery or ultrasound thermal therapy is that nanodroplets $(100-600 \mathrm{~nm}$ in diameter) cannot penetrate deep into the tumor tissue to reach the core after permeating across tumor's leaky vasculature. Therefore, their therapeutic utility in facilitating drug delivery or ultrasound thermal therapy by forming gas bubbles is greatly limited.

To overcome this major limitation, we started working on combining PFC-encapsulated nanodroplets with histotripsy, which is a new, non-invasive, image-guided, ultrasound ablation method [21-25]. Histotripsy uses extremely high pressure, microseconds long pulses to generate a cluster of microbubbles (bubble cloud) from pre-existing gas pockets in the tissue where the rapid expansion and collapse of the microbubbles disrupts cells $[22,23,25]$. We hypothesize that combining histotripsy with PFC-encapsulated nanodroplets will cause the ultrasound pulses to vaporize the nanodroplets delivered to the surface of the tumor nodules and form gas bubbles. Using the resulting gas bubbles as cavitation nuclei will significantly reduce the pressure threshold needed to generate and maintain the cavitation bubble cloud, which will fractionate the tumor nodules layer by layer until the entire tumor is destroyed. Whereas, the surrounding normal tissue without the nanodroplets will have a higher threshold and will be preserved from the histotripsy-induced fractionation. As such, even with nanodroplets diffusing only to regions next to tumor vasculature, targeted histotripsy will destroy the tumor core, overcoming the limitation caused by the shallow penetration depth of nanodroplets. This hypothesis is supported by our previous studies showing that once the cavitation bubble cloud is initiated, the histotripsy process can be maintained at a much lower pressure than that needed for initiation [24]. The ability of histotripsy alone to erode a tissue layer by layer has been demonstrated in excised tissue samples [26] and in vivo large animal models [27, 28]. Further, the resulting gas bubbles can function as ultrasound contrast agents, which will allow the tumor sites to be seen on ultrasound imaging and allow the histotripsy treatment to be guided and monitored in real-time by ultrasound imaging. By employing an ultrasound transducer with a large focal zone, this technique could also achieve efficient treatment of tumors with multiple nodules.

Before a nanodroplet-mediated histotripsy approach can be investigated in vivo, nanodroplets must be synthesized with an optimum size $(<500 \mathrm{~nm})$ that allows them to diffuse through the tumor vasculature [5-11]. Furthermore, these nanodroplets must be capable of reducing the pressure threshold to generate histotripsy bubbles and fractionate tissue. The goal of this study is to demonstrate the initial feasibility of nanodroplet-mediated histotripsy to set the foundation for future studies aimed at extending this technology to tumor ablation in vivo. We have synthesized an amphiphilic triblock copolymer that encapsulates PFC forming nanodroplets with an average diameter of $\sim 200 \mathrm{~nm}$. This ABC triblock copolymer is composed of a hydrophilic poly(ethylene glycol) (PEG) block, a central poly(acrylic acid) (PAA) block, and a random copolymer of heptadecafluorodecyl methacrylate (HDFMA) and methyl methacrylate (MMA) as the hydrophobic block. Nanodroplets are formulated using the $\mathrm{PEG}_{45}-b-\mathrm{PAA}_{10}-b-\mathrm{P}\left(\mathrm{HDFMA}_{8}-\mathrm{co}-\mathrm{MMA}_{21}\right)$ copolymer that encapsulates perfluoropentane (PFP, boiling temperature $29^{\circ} \mathrm{C}$ ) in the droplet's core. The PAA block is chemically cross-linked to "stitch" adjacent polymer chains forming a flexible polymer shell, which increases the stability of the formed nanodroplets. We hypothesized that our nanodroplets will remain stable at body temperature $\left(37^{\circ} \mathrm{C}\right)$ in the presence of serum proteins without an increase in their diameter above the size cutoff of tumor vasculature, which will allow them to extravasate into the tumor tissue in vivo.

In this report, we describe initial studies examining the ability to combine our nanodroplets with histotripsy to achieve efficient cell ablation in tissue-mimicking phantoms. We first simulated the peak negative pressure threshold needed to form a gas microbubble of $50 \mu \mathrm{m}$ in diameter using an initial bubble nucleus of 5-500 $\mathrm{nm}$ in diameter and a single 2-cycle pulse with different frequencies. In previous histotripsy studies, microbubbles were generated and expanded to $>50 \mu \mathrm{m}$ in diameter to produce cell disruption. This microbubble dynamic is different from other nanodroplet studies for targeted imaging and 
drug delivery, and therefore requires substantially different acoustic parameters. Based on the simulation results, a $500 \mathrm{kHz}$ focused transducer was used to apply a single 2-cycle ultrasound pulse, and the pressure threshold to acoustically vaporize the PFP encapsulated in the nanodroplet's core and expand the resulting gas bubble to $>50 \mu \mathrm{m}$ was measured. We also examined the feasibility of using this technique to produce cavitation in selected regions and image the resulting bubbles with ultrasound imaging. Finally, we evaluated the ability of nanodroplets to fractionate red blood cells embedded in tissue-mimicking agarose gels when combined with a histotripsy pulse.

\section{Experimental Section}

\section{Formulation and Characterization of Nanodroplets}

We synthesized an $A B C$ triblock copolymer composed of a hydrophilic poly(ethylene glycol) (PEG) block, a central poly(acrylic acid) (PAA) block, and a random copolymer of heptadecafluorodecyl methacrylate (HDFMA) and methyl methacrylate (MMA) as the hydrophobic block via a one-pot "click" reaction [29-31]. $\mathrm{PEG}_{45}-b-\mathrm{PAA}_{10}-b-\mathrm{P}\left(\mathrm{HDFMA}_{8}-\mathrm{co}-\right.$
$\mathrm{MMA}_{21}$ ) copolymers were used to prepare PFP-loaded nanodroplets following the procedure shown in Figure 1. Briefly, $\mathrm{PEG}_{45}-b-\mathrm{PAA}_{10}-b$ $\mathrm{P}\left(\mathrm{HDFMA}_{8}\right.$-co-MMA $\left.{ }_{21}\right)$ copolymers were dissolved in tetrahydrofuran (THF) $(0.2 \% \mathrm{w} / \mathrm{v})$ and cooled down to $0^{\circ} \mathrm{C}$ before the addition of PFP $(2 \% \mathrm{v} / \mathrm{v})$ while vigorously stirring the reaction mixture. An equal amount of water was slowly added to this solution mixture to trigger micelle formation and the mixture was stirred for $1 \mathrm{~h}$ in an ice bath. The micelles solution was transferred into a dialysis bag (MWCO of $1 \mathrm{kDa}$ ) and dialyzed against ice-cold 2-(N-morpholino) ethanesulfonic acid (MES) solution or water to remove the THF. After 12 hours of dialysis, a slightly milky solution of PFP-loaded nanodroplets was observed. The PAA block was chemically cross-linked using 2,2-(ethylenedioxy)-bis(ethylamine) as a cross-linker via NHS/EDC coupling chemistry [32-34] to form a flexible polymer shell that stabilizes the nanodroplets but allows acoustic droplet vaporization to form microbubbles. Selection of ideal polymer composition and PFP content used in this study has been optimized in a separate investigation $[30,31]$.

\section{Self-assembly of ABC Block Copolymer into Nanodroplets}

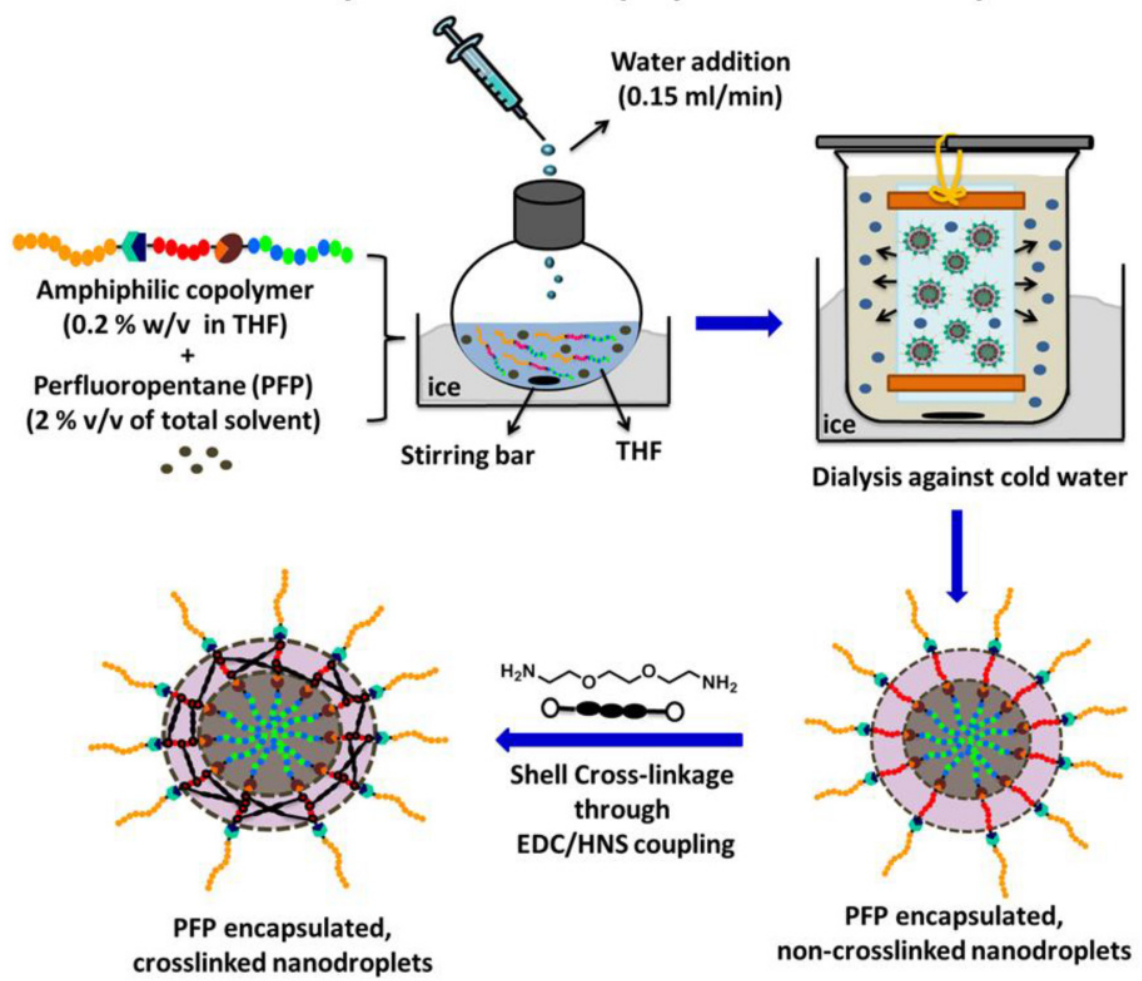

Fig I. Formulation of PFP-loaded nanodroplets by self-assembly of amphiphilic triblock $\mathrm{PEG}_{45}-b-\mathrm{PAA} \mathrm{A}_{10}-\mathrm{b}-\mathrm{P}\left(\mathrm{HDFMA}_{8}\right.$-co-MMA $\left.\mathrm{A}_{21}\right)$ copolymer around the PFP core followed by shell cross-linkage via EDC/HNS coupling reactions. 
Nanodroplet concentration and size distribution were characterized using Nanoparticle Tracking Analysis (NTA). Measurements were obtained using a NanoSight NS500 (NanoSight Ltd., Amesbury, United Kingdom), equipped with an LM14 (405 nm laser), high sensitivity EMCCD Andor Luca camera and a syringe pump. Upon dilution to the appropriate NTA concentration with DI Water (MilliQ, $18 \mathrm{M} \Omega$ ), both capture and analysis were carried out using the NTA software (Version 2.3, Build 0027) at $22^{\circ} \mathrm{C}$ and $37^{\circ} \mathrm{C}$. The samples were measured by capturing $60 \mathrm{~s}$ videos (6 videos per each sample). The error bars represent the standard deviation of the repeat measurements of each sample. The mean size and standard deviation values obtained by the NTA software correspond to arithmetic values calculated with the sizes of all particles analyzed for each sample $(n=6)$.

\section{Biocompatibility of Nanodroplets}

Toxicity of the $\mathrm{PEG}_{45}-b-\mathrm{PAA}_{10}-b-\mathrm{P}\left(\mathrm{HDFMA}_{8}-\mathrm{co}-\right.$ $\mathrm{MMA}_{21}$ ) copolymer used to formulate the nanodroplets was investigated as a function of polymer concentration using the MTT assay (EMD Millipore, Billerica, MA). Briefly, human prostate cancer cells (PC-3) were seeded at a seeding density of $1 \times 10^{5}$ cells/well in 96-well plates and allowed to adhere overnight before replacing the culture medium with phenol red-free RPMI 1640 medium solution containing $0.3,1.5,15,30$ and $75 \mu \mathrm{g} / \mathrm{mL}$ of the $\mathrm{PEG}_{45}-b-\mathrm{PAA}_{10}-b-\mathrm{P}\left(\mathrm{HDFMA}_{8}-\mathrm{co}-\mathrm{MMA}_{21}\right) \quad$ copolymer formulated into PFP-loaded nanodroplets with an average diameter of $\sim 200 \mathrm{~nm}$, which corresponds to droplets concentrations of $2.06 \times 10^{8}, 1.03 \times 10^{9}$, $1.03 \times 10^{10}, 2.06 \times 10^{10}, 5.15 \times 10^{10}$ particles $/ \mathrm{mL}$, respectively. The nanodroplets solution was aspirated after incubating for 24 hours under normal culture conditions followed by adding $10 \mu \mathrm{L}$ of the MTT reagent to each well and incubating for 2 hours according the manufacturer's guidelines to allow enough time for the formation of the purple formazan precipitate. A $100 \mu \mathrm{L}$ of the detergent solution was added to each well and incubated for 3 hours in the dark at room temperature before measuring the UV absorbance at $570 \mathrm{~nm}$ using a Multiskan microplate reader (Thermo Fisher Scientific Inc., Waltham, MA). PC-3 cells incubated with blank RPMI1640 culture medium and 5\% $\mathrm{v} / \mathrm{v}$ Triton X-100 solution were used as negative and positive controls, respectively. The statistical difference in UV absorbance between RPMI1640-treated cells (negative control) and those incubated with different concentrations of the copolymer was evaluated using student's $t$-test where $\mathrm{p}<0.05$ indicated a statistically significant difference in cell viability.

\section{Threshold Simulation for Nanodroplet- Mediated Cavitation}

A numerical simulation based on a modified Keller-Miksis equation for soft-tissue bubble dynamics $[35,36]$, was performed to estimate the relationship between frequency and bubble expansion for nanodroplet-mediated histotripsy with nanodroplets of 5-500 nm diameter. The peak negative pressure to expand initial bubbles of 5-500 $\mathrm{nm}$ diameters into 50 $\mu \mathrm{m}$ diameter bubbles was determined. Vaporization was not accounted for in this simulation, but the Pthresholds to achieve this bubble expansion are expected to be sufficiently high to vaporize the nanodroplets based on the literature $[12,37]$.

The simulation computed the bubble radius vs. time in response to a driving pressure waveform by

$$
\left(1-\frac{\dot{R}}{c}\right) R \ddot{R}+\left(1-\frac{\dot{R}}{3 c}\right) \frac{3}{2} \dot{R}^{2}=\left(1+\frac{\dot{R}}{c}\right) \frac{p_{w}(\dot{R}, R, t)}{\rho}+\frac{R}{\rho c} \frac{\partial p_{w}}{\partial t}
$$

where $R$ is the bubble radius, the dot indicates a derivative with respect to time, $c$ is the sound speed, $\rho$ is the medium density, and $p_{w}$ is the pressure at the bubble wall in the medium surrounding the bubble, defined as

$$
p_{w}(\dot{R}, R, t)=\left(p_{0}-p_{v}+\frac{2 \sigma}{R_{0}}\right)\left(\frac{R_{0}}{R}\right)^{3 \kappa}-p_{0}-\frac{2 \sigma}{R}-\frac{4 G}{3 R^{3}}\left(R^{3}-R_{0}^{3}\right)-\frac{4 \mu \dot{R}}{R}-p_{a}(t)
$$

In Eq. 2, $p_{0}$ and $p_{v}$ are the ambient and vapor pressure, respectively, $\sigma$ is surface tension, $R_{0}$ is the initial bubble radius, $\kappa$ is the polytropic index, $G$ is the shear modulus, $\mu$ is the dynamic viscosity, and $p_{a}(t)$ is the acoustic driving pressure. The tissue Young's modulus $(E \sim 3 G)$ and $\mu$ were chosen to match the agarose tissue phantom properties used in this study ( $E=38 \mathrm{kPa}$ and $\mu=0.05 \mathrm{~Pa}-\mathrm{s})$, which were within the property range of hepatocellular carcinoma $(E=$ $20.4-75 \mathrm{kPa})$, metastatic liver tumors $(E=23.6-75$ $\mathrm{kPa})$, and prostate cancer tissue $(E=24 \mathrm{kPa})[38,39]$. The surface tension $(\sigma)$ was estimated for soft tissue as

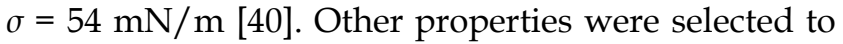
match values for water at $20^{\circ} \mathrm{C}$.

The pressure waveform for the simulation employed an analytical pulse model [41] to create a 2 -cycle, asymmetric pulse with a similar $P_{+} / P_{-}$to that in Figure 2. The pressure is given as

$$
p_{a}(t)=p_{f} \sum_{k=1}^{\infty}\left[\frac{\sin (k \omega(t-\tau)+\phi)}{k}\right] \cdot \exp \left[-\left(\frac{t-\delta}{\xi}\right)^{2}\right]
$$

where $p_{f}$ is the fundamental pressure amplitude, $\omega$ is the radial frequency, $\tau$ is the starting phase, $\phi$ is a phase shift to create waveform asymmetry, $\delta$ is the 
time delay to the center of the pulse, and $\xi$ defines pulse duration. The waveform at $0.5 \mathrm{MHz}, \omega=3.14 \mathrm{x}$ $10^{6} \mathrm{rad} / \mathrm{s}, \tau=1.25 \Pi / \omega, \phi=-\Pi / 4, \delta=6.3 \mu \mathrm{s}$, and $\xi=$ $2.6 \mu$ s. The simulation was also performed at $f=0.2$ and $1.1 \mathrm{MHz}$, scaling the above parameters to maintain the same waveform shape.

\section{Ultrasound Setup}

Ultrasound pulses were generated by two 500 $\mathrm{kHz}$ focused ultrasound transducers built in house. The two transducers were fabricated using rapid prototyping technology and contained 32 and 7 elements, respectively. Each module consists of a 2 inch $(50.8 \mathrm{~mm})$ diameter lead zirconate titanate (PZT) disc element coupled to an elliptical acoustic lens through a quarter wavelength matching layer. Transducer shells contained threaded receptacles populated with individual element modules. The 32 element transducer had an aperture diameter of $300 \mathrm{~mm}$, a focal distance of $150 \mathrm{~mm}$, and a focal zone (Measured -6 dB Pressure) of $1.8 \times 3.9 \mathrm{~mm}$. The 7 element transducer had an aperture diameter of $200 \mathrm{~mm}$, a focal distance of $150 \mathrm{~mm}$, and a focal zone (Measured $6 \mathrm{~dB}$ Pressure) of $3.4 \times 20.7 \mathrm{~mm}$. A field-programmable gate array (FPGA) was used as a custom pulse generator to electronically control each channel (Altera Corp., San Jose, CA). The FPGA was connected to a 32-channel bank of high voltage pulsers developed in-house.
Acoustic waveforms produced by the $500 \mathrm{KHz}$ therapeutic transducer (Figure 2A) were obtained using a fiber optic probe hydrophone built in house [42]. Pressure wave measurements were recorded in free-field in degassed water at room temperature. At higher pressures above the histotripsy cavitation threshold, pressure could not be accurately measured due to instantaneous cavitation at the fiber tip. These higher pressure levels were estimated as a summation of the pressures generated by the individual elements, which were sparsely distributed without a significantly overlapping beam and closely matched the predicted values using a linear extrapolation of the lower pressure values measured with all elements. The 7 element transducer was used for the selective cavitation generation experiment, because the larger focal region of this transducer was needed for that experiment set. The 32 element transducer was used for all other experiments, as it is capable of generating high pressure beyond the intrinsic threshold. Cavitation bubbles were optically imaged using a high-speed, 1 megapixel CCD camera, (Phantom V210 - Vision Research, Wayne, New Jersey, USA) (Figure 2B). The camera was focused through a macro lens (Tominon $105 \mathrm{~mm} \mathrm{f} / 4.5$ Kyocera Corp., Japan) and folding optical bellows (Nikon Corp., Japan).

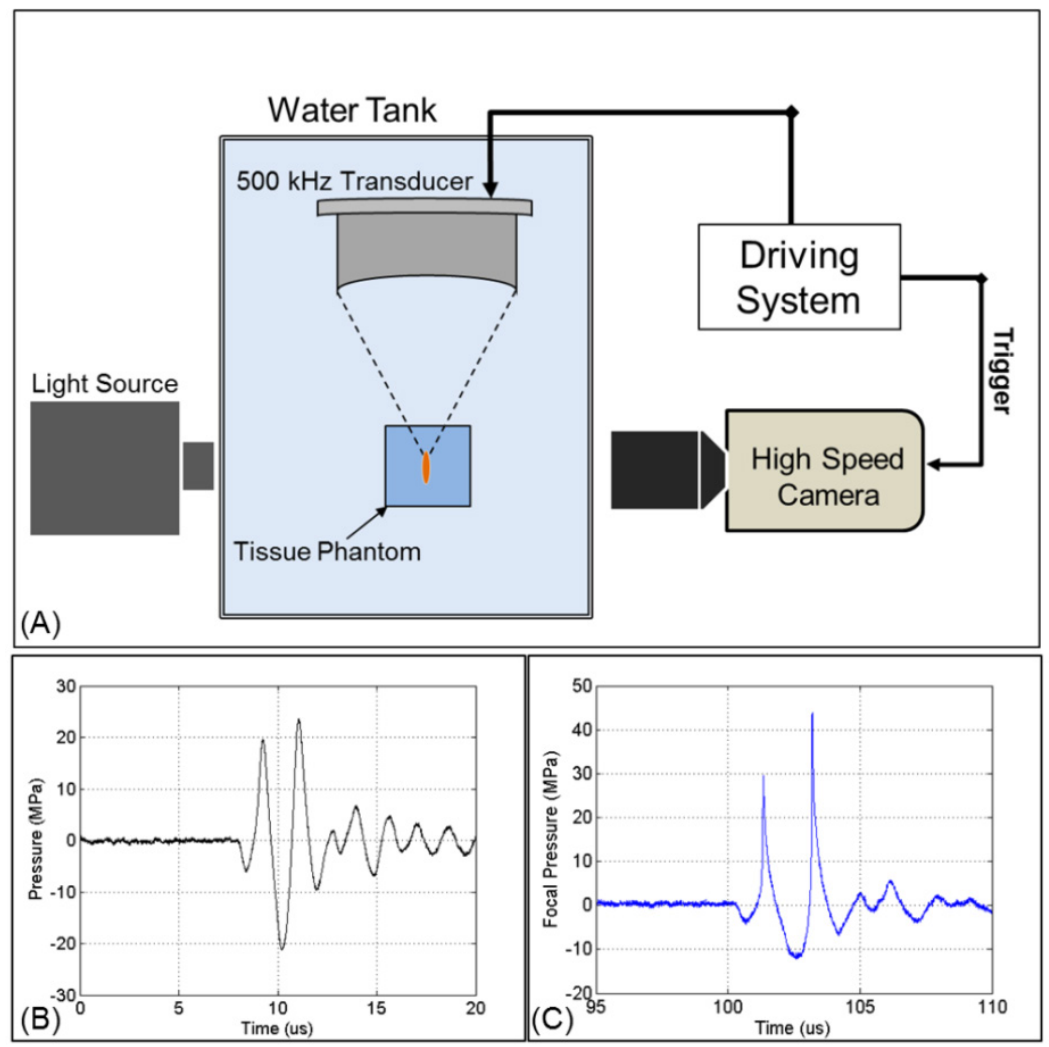

Fig 2. Acoustic waveform and experimental setup. (A) The focus of one of the $500 \mathrm{kHz}$ transducers was aligned inside tissue phantoms containing nanodroplets. Cavitation was monitored using high speed optical imaging. Example of a 2-cycle histotripsy pulse generated by the (B) 32 element and (C) 7 element transducers. 


\section{Preparation of Tissue Phantoms}

Agarose phantoms were used to provide a well-controlled viscoelastic medium for this study, as histotripsy-induced damage is highly dependent on the tissue mechanical properties. Tissue phantoms containing $1 \%$ agarose $\mathrm{w} / \mathrm{v}$ were prepared by slowly mixing agarose powder (Agarose Type VII, Sigma-Aldrich, St. Louis, MO) into saline solution $(0.9 \%$ sodium chloride, Hospira) heated to above $70^{\circ} \mathrm{C}$ while stirring until the gel became completely transparent. Agarose solutions were degassed under a partial vacuum of $20.5 \mathrm{mmHg}$ for 30 minutes before allowing the agarose solutions to cool down to $37^{\circ} \mathrm{C}$. Phantoms containing nanodroplets were prepared by slowly adding the nanodoplets $\left(2.36 \times 10^{8}\right.$ particles $\left./ \mathrm{mL}\right)$ into the agarose solution while stirring. The agarose mixtures were poured into rectangular polycarbonate holders and placed in a refrigerator at $4^{\circ} \mathrm{C}$ to allow the solution to solidify forming tissue phantoms with embedded nanodroplets (test) or without nanodroplets (control). The Young's modulus of the tissue phantom was $38 \mathrm{kPa}$ [43], which is within the range of the Young's modulus of hepatocellular carcinoma tumors $(20.4-75 \mathrm{kPa})$, metastatic liver tumors $(23.6-75 \mathrm{kPa})$, and prostate tumors $(24 \mathrm{kPa})[38,39]$. Tissue phantoms were warmed prior to use and maintained at $37^{\circ} \mathrm{C}$ for the duration of all experiments performed in this work.

For cell fractionation experiments, tissue phantoms with a red blood cell (RBC) layer were prepared using canine RBCs in $0.9 \%$ isotonic saline and $1 \%$ agarose solutions with and without nanodroplets. Fresh canine blood was obtained from adult research subjects in an unrelated study and added to an anticoagulant solution of citratephosphate-dextrose (CPD) (Sigma-Aldrich Co., St. Louis, MO) with a CPD-to-blood ratio of 1:9 mL. Whole blood was separated in a centrifuge at $3000 \mathrm{rpm}$ for $10 \mathrm{~min}$. The plasma and white buffy coat were removed, and the RBCs were saved for addition to the phantom. To prepare the RBC phantom, an initial layer of $1 \%$ agarose mixture (with and without nanodroplets) was poured into a rectangular polycarbonate housing to fill half of it at $37^{\circ} \mathrm{C}$. The housing was placed in a refrigerator at $4^{\circ} \mathrm{C}$ to allow the agarose to cool and solidify. The remaining solution was kept at $37^{\circ} \mathrm{C}$. A small amount of agarose solution was mixed with the RBCs (5\% RBCs v/v). The frame with solidified agarose was removed from refrigeration, and a thin layer of the RBC-agarose solution was poured onto the gel surface to allow the entire surface to coat in a thin layer. After $5 \mathrm{~min}$, the RBC-agarose layer was solidified, and the remaining agarose solution without RBCs was poured to completely fill the frame. This procedure created a thin layer of RBCs suspended in the center of the agarose phantom.

\section{Measurement of Histotripsy Threshold in Agarose Tissue Phantoms}

The pressure threshold to form and expand microbubble to $>50 \mu \mathrm{m}$ diameter using a single 2-cycle ultrasound pulse was recorded in agarose tissue phantoms with and without nanodroplets. To determine the cavitation threshold, the ultrasound pulse was applied to the center of tissue phantoms with and without nanodroplets using the 32 element $500 \mathrm{kHz}$ transducer at pressures ranging from $2-40 \mathrm{MPa}$. The sample size was 100 pulses for each pressure level by applying 100 pulses separated by $10 \mathrm{sec}$ between pulses (i.e., pulse repetition frequency (PRF) of 0.1 $\mathrm{Hz}$ ), to provide adequate time for any residual nuclei from the bubble collapse by the previous pulse to dissolve. In this way, each of the 100 pulses was expected to generate cavitation independently without influence from the previous pulses. To evaluate the threshold variation using different nanodroplet formulations in different phantoms, this process was repeated using nanodroplets synthesized on 3 different occasions each evaluated using two tissue phantoms (total $n=6$ ) using 100 pulses at each pressure level.

Cavitation was monitored using high-speed optical imaging (Figure 2). A high-speed V210 camera (Vision Research, Wayne, NJ) was used to capture images of the focal zone directly after the propagation of each pulse through the focus in the phantoms. The camera was focused with a macro-bellows lens (Tominon, Kyocera, Kyoto, Japan) through the optical window of the chamber to observe the focal region. The camera was backlit by a continuous light source to produce shadowgraphic images of cavitation bubbles. The camera was triggered to record one image for each pulse applied, 10-40 $\mu$ s after the beginning of the pulse reached the focal center. The timing was actively adjusted at each pressure level to coincide with the maximal bubble expansion occurrence. This process ensured that the pressure pulse had passed over the entire focal volume at the time of capture and the bubbles were imaged at the point of maximal expansion. The camera exposure time was $2 \mu$ for all images. After acquisition, images were converted from grayscale to binary by an intensity threshold determined based on the background intensity of the shadowgraphs in image-processing software (Matlab; The Math-Works, Natick, MA, USA). The events when a bubble was expanded to $>50 \mu \mathrm{m}$ within the focus were analyzed and recorded for each pressure level because such bubble expansion was shown in histotripsy to achieve tissue fractionation. The num- 
ber of pulses (out of 100) in which one or more such cavitation events was observed to occur was recorded for each sample as the cavitation probability $\left(\mathrm{P}_{\text {cav }}\right)$. The average cavitation probability at each pressure level for the six samples was plotted for tissue phantoms containing nanodroplets as well as control phantoms without nanodroplets with the error bars representing the standard deviation. An interpolation fit was performed on the plot in Matlab to calculate the P-threshold, which was considered to be the point at which $50 \%$ of the pulses generated cavitation bubbles $>50$ um (i.e. $P_{\text {cav }}=0.50$ ). The $\mathrm{P}$ - threshold was calculated for each sample $(n=6)$ and reported as the mean \pm the standard deviation. The minimum pressure level at which any cavitation bubbles $>50 \mu \mathrm{m}$ were observed was similarly analyzed and reported as the mean \pm the standard deviation. Finally, the difference between $P_{\text {cav }}=0.15$ and $P_{\text {cav }}=0.85$, a relative measure of the steepness of the transition from $P_{\text {cav }}=0$ to $P_{\text {cav }}=1$ was calculated and reported as the mean \pm the standard deviation for phantoms containing nanodroplets as well as histotripsy only controls.

\section{Selective Cavitation in Composite Agarose Phantom}

Using a focal zone covering multiple sub-regions loaded with the nanodroplets adjacent to other sub-regions without the nanodroplets, we hypothesized that cavitation can be selectively generated only in the sub-regions containing the nanodroplets by applying ultrasound at an acoustic pressure that is above the cavitation threshold with nanodroplets but below the cavitation threshold using histotripsy alone. To test this hypothesis, we prepared agarose phantoms containing hexagonal inclusions that were the only regions within the gel loaded with the nanodroplets. Briefly, composite tissue phantoms were prepared by pouring $1 \%$ agarose solution into a rectangular polycarbonate housing before submerging and fixing eight $6.5 \mathrm{~mm}$ thick hexagonal inclusions inside the phantom. The phantom with the inclusions was placed in a refrigerator at $4^{\circ} \mathrm{C}$ to allow the agarose to solidify. After the agarose solution solidified, the hexagon inclusions were removed from the tissue phantom leaving hexagonal voids that were then filled with $1 \%$ agarose solution containing the nanodroplets at a concentration of $3.0 \times 10^{8}$ particles $/ \mathrm{mL}$. This procedure created a tissue phantom with hexagonal "islands" containing nanodroplets surrounded by an agarose gel without nanodroplets. Subsequently, the focus of the 7 element transducer was aligned to cover two of the nanodropletscontaining hexagons and the agarose gel without nanodroplets between them. Histotripsy pulses were applied to the focus at a PRF of $10 \mathrm{~Hz}$ at a peak nega- tive pressure of 17.3 MPa. The resulting cavitation bubbles were monitored by high-speed optical imaging. Additionally, cavitation bubble generation was monitored by a clinical ultrasound imaging system (HDI 5000 Ultrasound system).

\section{Bubble Behavior}

To determine if nanodroplet-mediated cavitation bubbles exhibited similar expansion and collapse behavior to histotripsy bubbles, optical images of the growth and collapse of bubbles were recorded by the high-speed camera. Nanodroplet-mediated cavitation bubbles were generated by the 32 element $500 \mathrm{kHz}$ transducer at a peak negative pressure of $15.6 \mathrm{MPa}$. This pressure level was selected because it is lowest pressure at which a cavitation cloud was always generated using a 2-cycle ultrasound pulse in tissue phantoms with nanodroplets. The expansion and collapse behavior of bubbles was reconstructed from a series of bubble clouds imaged at different time delays after the pulse. Ten pulses were applied to agarose tissue phantoms containing nanodroplets at each time delay at a PRF of $1 \mathrm{~Hz}$. The focus was then moved to a different location within the tissue phantom and camera delay was increased $3 \mu \mathrm{s}$. Delay intervals of 0-60 $\mu$ s were used to reconstruct a sequence of bubble images covering the entire period of bubble expansion and collapse. Shadowgraph images were converted from grayscale to binary by an intensity threshold determined by the background intensity using image processing software (Matlab, The Mathworks). Bubbles were indicated by black regions $>5$ pixels. The minimum resolvable diameter of a bubble using this minimum 5-pixel area was approximately $15 \mu \mathrm{m}$. The average diameter of $10 \mathrm{bub}-$ bles visible within the focus was measured for each pulse at each time delay. A sample size of 10 pulses was used for each time delay and the average bubble size \pm the standard deviation was plotted as a function of time.

\section{Ablation of Red Blood Cells in Tissue Phantom}

Agarose gel phantoms with an embedded RBC layer were used to characterize cell fractionation induced by nanodroplet-mediated histotripsy. Fractionation of the RBCs turns the color of the embedded cell layer from opaque red to translucent as the RBCs were lysed, which allows direct visualization of the histotripsy-induced fractionation process [44]. Previous studies have also shown that the lesion visualized in RBC phantoms is similar to the lesion generated in tissue identified by histology [44]. The focus of the 32 element $500 \mathrm{kHz}$ transducer was aligned with the center of the red blood cell phantom layer and 2000 histotripsy pulses were applied at a PRF of $10 \mathrm{~Hz}$ at 
peak negative pressures of $6.2 \mathrm{MPa}, 11.0 \mathrm{MPa}, 15.9$ $\mathrm{MPa}$, and $20.7 \mathrm{MPa}$ with and without nanodroplets $(n=6)$. The bubble cloud and resulting cell fractionation was recorded by high-speed optical imaging after each pulse (Figure 2). The high-speed camera (Phantom V211, Vision Research) was focused to the red blood cell layer and backlit by a continuous light source. The camera was triggered to record two images for each applied pulse, one $10 \mu$ s after the pulse reached the focus to visualize the bubble activity and another frame between pulses, $50 \mathrm{~ms}$ after each pulse, to assess tissue damage. The camera exposure time was $10 \mu \mathrm{s}$. The bubbles appeared as black regions in the shadowgraphic image and RBC fractionation was visualized as RBCs turn transparent after fractionation. Cell fraction was compared between RBC phantoms with nanodroplets and control RBC phantoms without nanodroplets. Shadowgraph images were converted from grayscale to binary by an intensity threshold determined by the background intensity using image processing software (MATLAB, The Mathworks) and the lesion area after 2000 pulses was calculated by summing the pixels within the fractionated region.

\section{Results and Discussion}

\section{Nanodroplets Formulation and Biocompati- bility}

Results show that $\mathrm{PEG}_{45}-b-\mathrm{PAA}_{10}-b$ $\mathrm{P}\left(\mathrm{HDFMA}_{8}-\mathrm{co}-\mathrm{MMA}_{21}\right)$ copolymer successfully encapsulated PFP $(2 \% \mathrm{v} / \mathrm{v})$ forming nanodroplets with an average diameter of $192 \pm 4.6 \mathrm{~nm}$ and $204 \pm 4.7 \mathrm{~nm}$ at $22^{\circ} \mathrm{C}$ and $37^{\circ} \mathrm{C}$, respectively. Figure $3 \mathrm{~A}$ is a representative image showing the size distribution for a single sample of nanodroplets in the range of 100-400 nm with $>99 \%$ of the nanodroplets smaller than 400 $\mathrm{nm}$ and $<0.01 \%$ larger than $600 \mathrm{~nm}$. The peak of the size distribution is $128.5 \pm 9.1 \mathrm{~nm}$ and $138.5 \pm 8.0 \mathrm{~nm}$ at $22^{\circ} \mathrm{C}$ and $37^{\circ} \mathrm{C}$, respectively. The similar size distribution at $22^{\circ} \mathrm{C}$ and $37^{\circ} \mathrm{C}$ indicates that the encapsulated PFP core remained as a liquid at the body temperature $\left(37^{\circ} \mathrm{C}\right)$ and did not change into a gas, which is consistent with previous studies [12-14]. The observed control over the size and shape of the formed nanodroplets is a result of the fine control over the length, molecular weight, and composition of the amphiphilic polymer used to formulate these nanodroplets $[45,46]$. Specifically, the PAA and P(HDFMA-co-MMA) blocks in the amphiphilic $\mathrm{PEG}_{45}-b$-PAA ${ }_{10}-b-\mathrm{P}\left(\mathrm{HDFMA}_{8}-\mathrm{co}-\mathrm{MMA}_{21}\right) \quad$ copolymer were synthesized via atom transfer radical polymerization (ATRP), which allows fine control over the length, composition, and functionality of the formed blocks [45] [47].
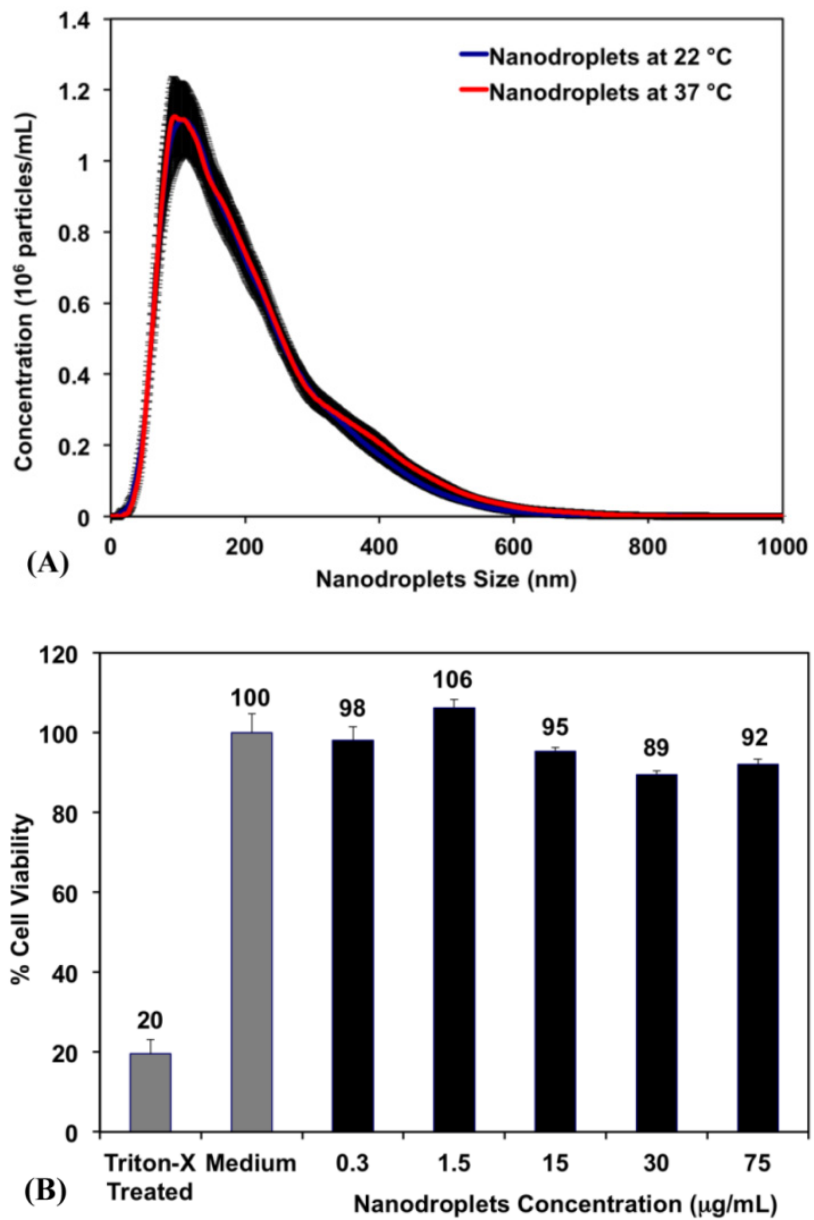

Fig 3. (A) A representative image showing the size distribution of PFP-loaded nanodroplets. Results show an average nanodroplet size of approximately $200 \mathrm{~nm}$ with the peak of the size distribution at $128.5 \pm 9.1$ $\mathrm{nm}$ and $138.5 \pm 8.0 \mathrm{~nm}$ at $22^{\circ} \mathrm{C}$ and $37^{\circ} \mathrm{C}$, respectively. (B) MTT results show no significant change in the viability of PC-3 cells incubated with 0.3 , I. $5,15,30$ and $75 \mu \mathrm{g} / \mathrm{mL}$ of the triblock copolymer used to formulate the PFP-loaded nanodroplets compared to PC-3 cells incubated with blank culture medium.

Research showed that self-assembled polymeric micelles tend to disassemble at higher temperatures and upon dilution to concentrations below their critical micelle concentration [45]. Nanodroplets with polymer, lipid, and protein shells have also been shown to coalesce forming larger microbubbles, which limits their ability to diffuse across tumor vasculature in vivo [48]. Therefore, we relied on covalent chemical cross-linkage of the polymer shell to increase nanodroplets stability against physiological shear stress in vivo and maintain the size, shape and concentration of the nanodroplets. Our results show that nanodroplets' concentration at $22^{\circ} \mathrm{C}\left(3.07 \times 10^{10}\right.$ particles $/ \mathrm{mL}$ ) did not change upon increasing the solution temperature to $37^{\circ} \mathrm{C}\left(3.00 \times 10^{10}\right.$ particles $\left./ \mathrm{mL}\right)$ (Figure 3A), which is critical for the success of the envisioned ablation approach as the number of cavitation nuclei is directly proportional to the number of stable nanodroplets present at tumor site. The initial testing 
of stability and biocompatibility of these nanodroplets is promising. Further, the size range of our nanodroplets should allow them to preferentially diffuse through the leaky tumor vasculature while preventing diffusion through normal blood vessels [1-4]. In addition, this nanodroplet design allows the covalent attachment of different targeting ligands to the free end of the PEG block allowing the incorporation of a "tunable" number of targeting motifs on the droplets surface. These targeted nanodroplets will allow selective binding to cancer cells, which should increase droplets concentration and retention in the tumor tissue.

Research shows that $90 \%$ of the PFP concentration in the blood can be eliminated from the body within 10 minutes after their administration, which diminishes the risk of toxicity [49]. Therefore, we investigated the toxicity of the $\mathrm{PEG}_{45}-b-\mathrm{PAA}_{10}-b$ $\mathrm{P}\left(\mathrm{HDFMA}_{8}-\mathrm{co}-\mathrm{MMA}_{21}\right)$ polymer used to formulate the nanodroplets as a function of polymer concentration using the MTT assay. Results show that there was no change in the number of viable PC-3 cells upon incubation with the PFP-loaded nanodroplets for 24 hours up to a polymer concentration of $75 \mu \mathrm{g} / \mathrm{mL}$ (5.15x $10^{10}$ droplets $/ \mathrm{mL}$ ) compared to the negative control $(p>0.05)$ (Figure 3B). This is a significant finding given that the highest reported nontoxic concentration of nanodroplets that were formulated using PEG-lipid conjugates is $2.5 \times 10^{8}$ droplets/mL[50], which is 200 -fold lower than the nanodroplets concentration used in our study. These results collectively indicate the ability to reproducibly formulate stable nanodroplets that are nontoxic to mammalian epithelial cells at high concentrations up to $5.15 \times 10^{10}$ particles/mL.

\section{Threshold Simulation for Nanodroplet- Mediated Cavitation}

In a previous histotripsy study, the threshold to generate cavitation using a single, $\mu$ s-length pulse in multiple tissues and tissue-mimicking media was measured to be 26-30 MPa peak negative pressure [36]. At the high pressure above $26 \mathrm{MPa}$, cavitation bubbles are generated from pre-existing gas pockets in the tissue $<10 \mathrm{~nm}$ in size and expanded to $>50 \mu \mathrm{m}$ followed by energetic collapse, all within a hundred $\mu \mathrm{s}$. This energetic bubble activity disrupts adjacent cells and eventually produces complete fractionation of cells in the target tissue into a liquefied homogenate. To determine the effects of nanodroplets on reducing the peak negative pressure (P-) threshold to generate and expand microbubbles to $>50 \mu \mathrm{m}$, a numerical simulation based on a modified Keller-Miksis equation for soft-tissue bubble dynamics as outlined in a previous study [36] was performed using initial gas bubbles 5-500 $\mathrm{nm}$ in diameter. We will refer to this threshold as the "histotripsy threshold" in this paper to distinguish from cavitation threshold commonly referred in the literature, which is often observed to be at much lower pressure levels with the presence of nuclei on the order of microns and/or using much longer pulses [51, 52]. The simulation used a 2-cycle pulse and compared frequencies of 0.2 , 0.5 , and $1.1 \mathrm{MHz}$. The simulation showed that the histotripsy threshold increased substantially with increasing frequency (Figure 4). For example, the histotripsy threshold using an initial $200 \mathrm{~nm}$ bubble was $1.7,6.3$, and $21.3 \mathrm{MPa}$ at $0.2,0.5$, and $1.1 \mathrm{MHz}$, respectively. Using an initial gas bubble at a diameter of $200 \mathrm{~nm}$ and above, there was no significant change in the threshold for the same frequency, whereas using a $100 \mathrm{~nm}$ initial nucleus, the histotripsy threshold was higher. The initial bubble size mattered little in the $200-500 \mathrm{~nm}$ range because the pressure amplitude to achieve $50 \mu \mathrm{m}$ max radius is much larger than the inertial cavitation threshold for bubbles this size. In this case the bubble motion is dominated by inertia, which leads to bubbles expanding to approximately the same size regardless of initial radius. Nanodroplet vaporization was not accounted for in this simulation, but $\mathrm{P}$ - thresholds are expected to be sufficiently high to vaporize the nanodroplets based on the literature $[12,37]$.

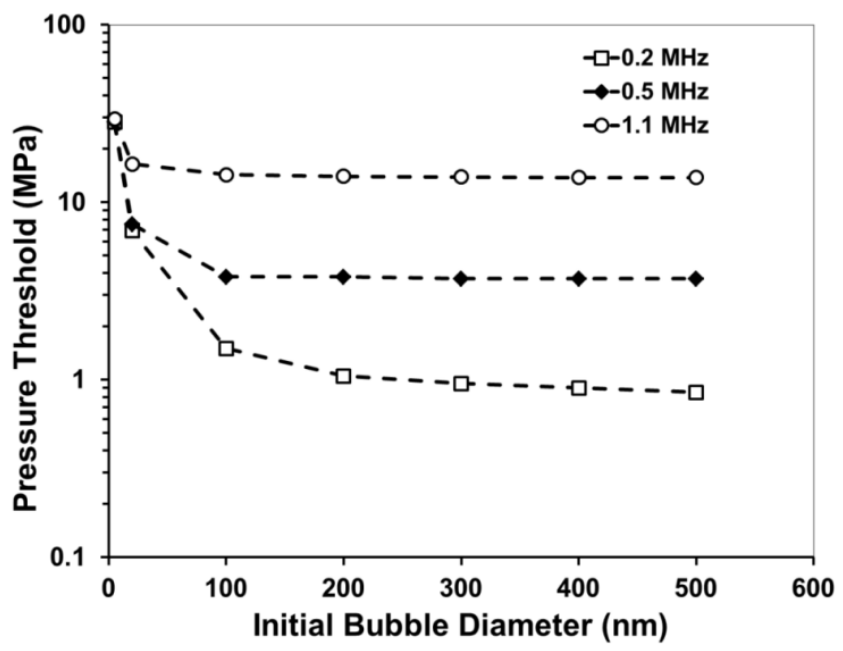

Fig 4. A plot showing the simulated peak negative pressure (P-) threshold to expand a bubble from an initial diameter of $5-500 \mathrm{~nm}$ to $50 \mu \mathrm{m}$ in tissue.

These results suggest that a lower frequency $(\leq 500 \mathrm{kH})$ would be better suited to use for nanodroplet-mediated histotripsy, as it results in a significantly lower histotripsy threshold. This dependence is opposite to a previous study by Kripfgans et al. using micron-sized droplets, where they found the pressure threshold to vaporize the droplets decreased with increasing frequency [13]. In compari- 
son, we used much smaller droplets and measured the threshold to expand the bubbles resulting from vaporized droplets to $>50 \mu \mathrm{m}$. It is likely that a different mechanism is responsible for our study. The trend of decreasing threshold with decreasing frequency is consistent with the frequency dependency in inertial cavitation, which we plan to investigate as a potential mechanism for nanodroplet-mediated histotripsy.

Using a lower frequency offers multiple advantages for ablation therapy. First, as the transducer focal zone scales with the wavelength, a low frequency transducer will yield a large focal zone, allowing histotripsy to be applied simultaneously to cover multi-nodule tumors seeded with our nanodroplets, thus increasing the treatment efficiency for multiple tumor nodules. With the reduced threshold at the tumor nodules with nanodroplets, only tumors will be treated while preserving surrounding normal tissue. Lower frequency is also more resistant to acoustic aberration and attenuation from bone obstruction and long overlying tissue, resulting in deeper penetration depth.

\section{Measurement of Histotripsy Threshold in Agarose Tissue Phantoms}

In order to validate the simulation results, the pressure threshold to vaporize the nanodroplets and expand the resulting bubbles $>50 \mu \mathrm{m}$ was measured in tissue-mimicking agarose phantoms with and without nanodroplets. Based on the analysis of high-speed images, results demonstrated a significant decrease in the histotripsy threshold with nanodroplets compared to histotripsy only controls. The minimum pressure level at which cavitation bubbles $>50 \mu \mathrm{m}$ was observed by high speed images to be $3.02 \pm 2.49 \mathrm{MPa}$ for phantoms containing nanodroplets and $15.60 \pm 2.35 \mathrm{MPa}$ for histotripsy only controls without nanodroplets (Figure 5). The pressure level at which $50 \%$ of the 100 pulses generated cavitation bubbles $>50 \mu \mathrm{m}, \mathrm{P}_{\text {cav }}=0.50$, was found to be $10.77 \pm 0.32 \mathrm{MPa}$ with nanodroplets compared to $28.79 \pm 0.47 \mathrm{MPa}$ without nanodroplets $(n=6)$. The difference between $P_{\text {cav }}=0.15$ and $P_{\text {cav }}=0.85$ was found to be $3.17 \pm 0.65 \mathrm{MPa}$ and $2.14 \pm 0.41 \mathrm{MPa}$ for phantoms with and without nanodroplets, respectively. At pressures $\geq 15.9 \mathrm{MPa}$, cavitation microbubbles were always observed $\left(\mathrm{P}_{\text {cav }}=1\right)$. At pressure greater than $11 \mathrm{MPa}$, the area of the bubble cloud increased with increasing pressure (Figure 6). For example, the lateral width of the bubble cloud was $1.1 \mathrm{~mm}, 2.0 \mathrm{~mm}$, and $2.4 \mathrm{~mm}$ at peak negative pressures of $13.9 \mathrm{MPa}$, 15.9 $\mathrm{MPa}$, and $20.7 \mathrm{MPa}$, respectively. These widths match closely with the lateral width of the focal zone where the pressure was above $11 \mathrm{MPa}$.

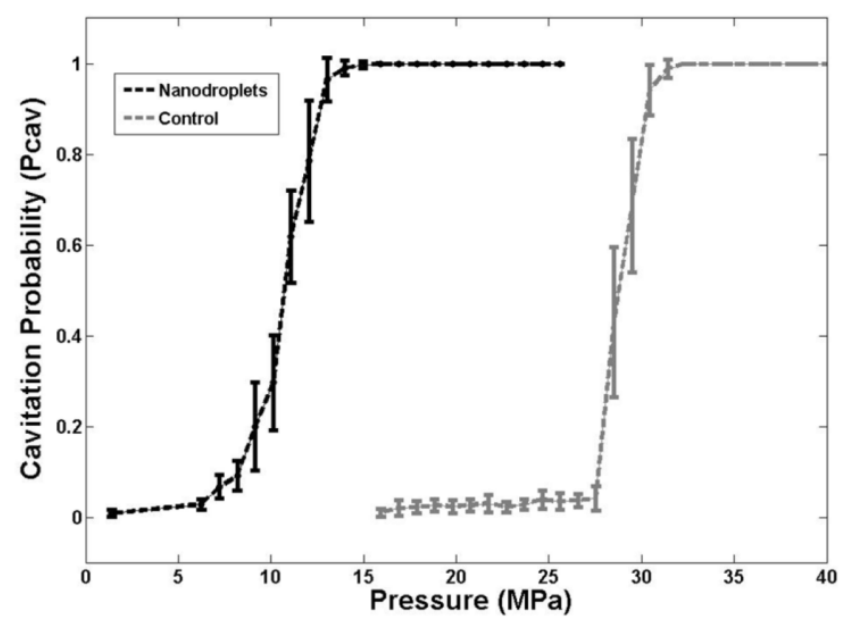

Fig 5. A plot showing the histotripsy threshold in agarose tissue phantoms with and without nanodroplets. Results demonstrated a significant decrease in the pressure threshold to generate cavitation bubbles $>50 \mu \mathrm{m}$ with nanodroplets compared to control conditions (histotripsy only).

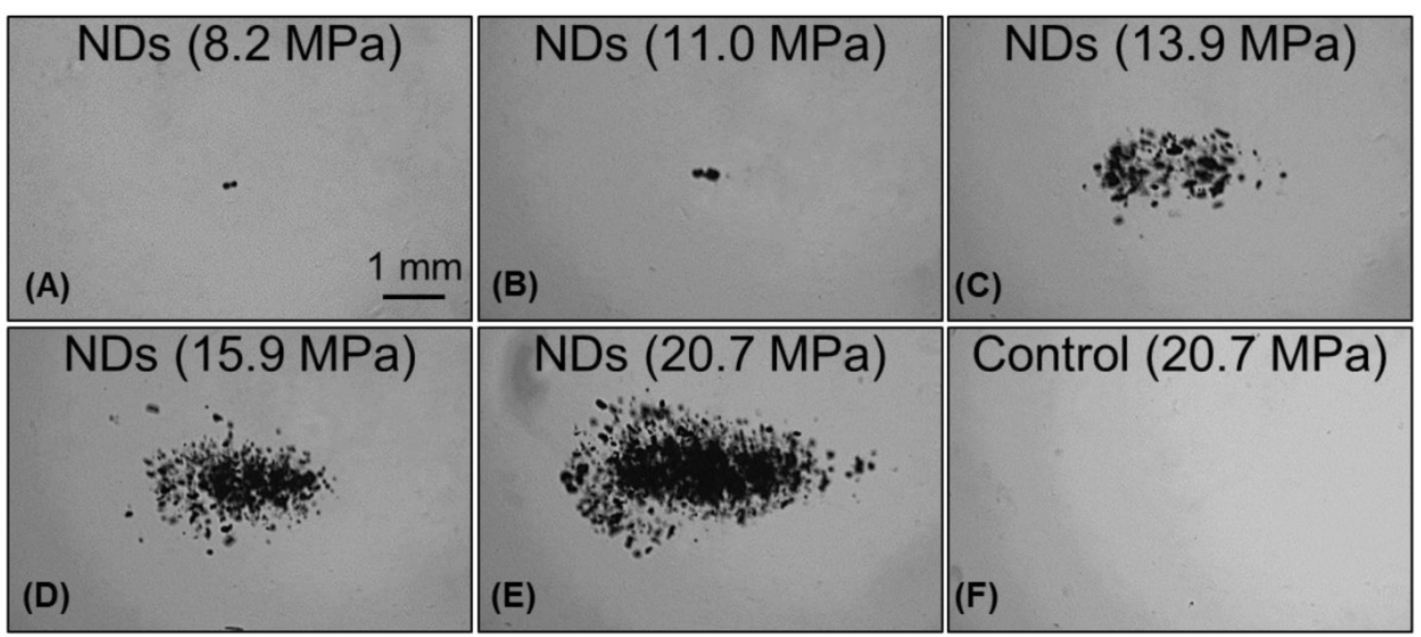

Fig 6. Images of nanodroplet-mediated cavitation in agarose tissue phantoms at increasing acoustic pressures. Images of cavitating bubble clouds (dark) generated using agarose phantoms containing nanodroplets at the pressure of 8.2-20.7 MPa (A-E). With increasing pressure, the area of the bubble cloud increased. In comparison, cavitation wasn't observed in control samples in this pressure range MPa (F). 
The experimental results further support our hypothesis that histotripsy-induced cavitation bubble cloud can be formed at a significantly reduced pressure using nanodroplets, which is the basis to achieve selective tumor ablation using nanodroplet-mediated histotripsy. These experimental results also match well with the simulation. The simulation results showed a $200 \mathrm{~nm}$ initial nucleus and a single $500 \mathrm{kHz}$ ultrasound pulse results in a histotripsy threshold of $6.3 \mathrm{MPa}$. Measured with the $500 \mathrm{kHz}$ transducer and $200 \mathrm{~nm}$ nanodroplets, the histotripsy threshold was $9.01 \pm 0.62 \mathrm{MPa}$ at $\mathrm{P}_{\text {cav }}=0.15$ and $10.8 \pm 0.3 \mathrm{MPa}$ at $\mathrm{P}_{\text {cav }}=$ 0.5 . The measured threshold was higher likely because droplet vaporization and the effects of the cross-linked polymer shell were not included in the simulation. Although the vaporization threshold wasn't measured in this work, the pressures used here are higher than the ADV threshold using similar sized nanodroplets reported in the literature [12-14]. While the pressure required to generate histotripsy bubbles using these nanodroplets was significantly lower than histotripsy alone, it is possible that the histotripsy threshold could be further reduced by using a lower boiling temperature PFC core, such as decafluorobutane (boiling temperature $-1^{\circ} \mathrm{C}$ ), which have been shown to vaporize at significantly lower pressure [14].

\section{Selective Cavitation in Composite Agarose Phantom}

Based on the reduced histotripsy threshold using nanodroplets, cavitation should be selectively generated in multi-tumor nodules with nanodroplets inside the focal zone, whereas no cavitation should be formed at normal tissue without nanodroplets. The feasibility of using nanodroplets to generate selective cavitation was investigated using a composite agarose tissue phantom. Histotripsy pulses were applied inside the composite agarose tissue phantom containing inclusions with nanodroplets. Using 2-cycle pulses at a peak negative pressure of $17.3 \mathrm{MPa}$, which is above the histotripsy threshold using nanodroplets and below the intrinsic threshold using histotripsy alone, cavitation bubble clouds were formed only in the inclusions containing nanodroplets located within the focal volume of the $500 \mathrm{kHz}$ transducer (Figure 7A). Optical images indicated two separate dense cavitation clouds inside the nanodroplet-containing inclusions inside the focal region throughout the 2000 pulses while no bubbles were observed outside the inclusions within the focal region (Figure 7B). Correspondingly, ultrasound imaging showed two dynamically changing hyperechoic zones inside the hexagon inclusions, while no bubbles were observed elsewhere (Figure 7C). As these cavitation bubbles generated in the process are viewed clearly on ultra- sound imaging, the histotripsy therapy process can be monitored and guided by ultrasound imaging in real-time.
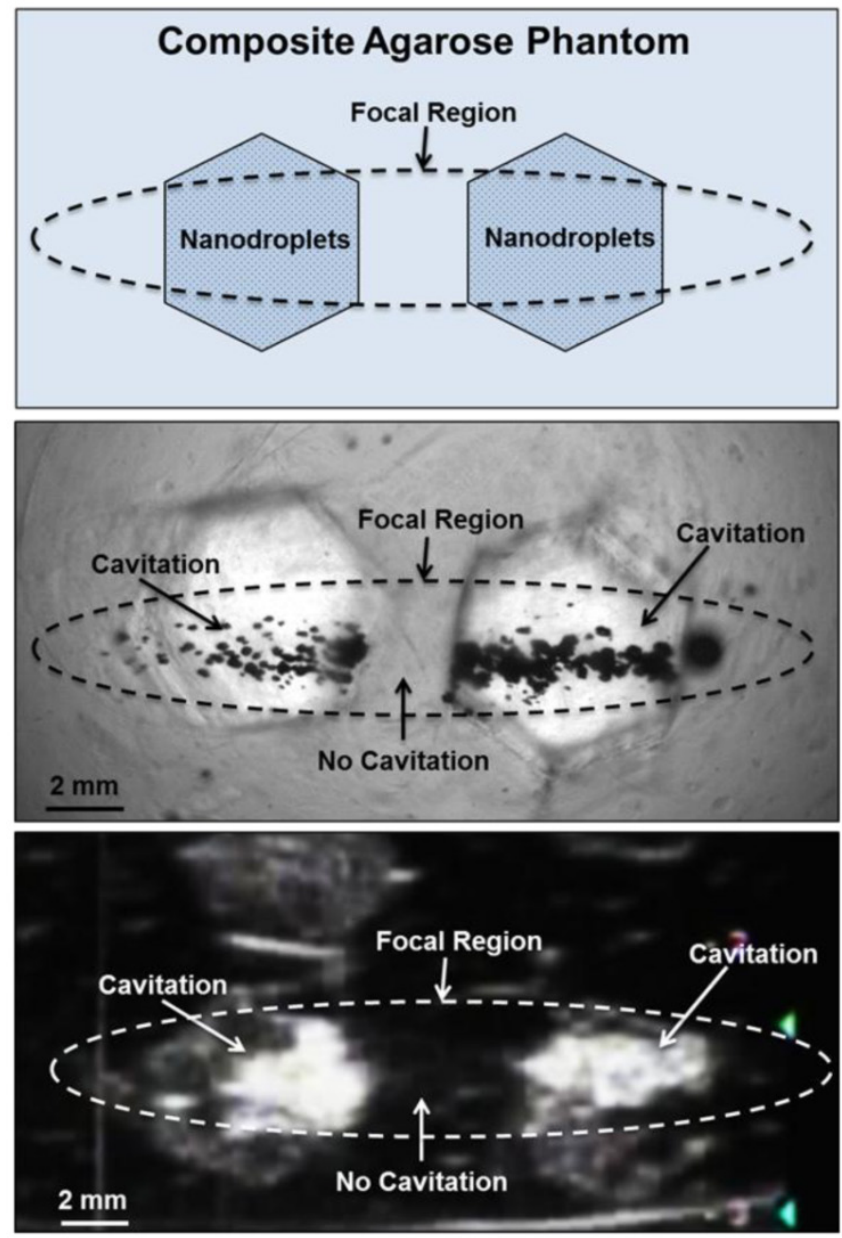

Fig 7. Selective cavitation in a composite agarose tissue phantom. Optical images (top) of agarose phantoms with hexagon shaped inclusions containing nanodroplets demonstrated cavitation within the focal area only occurred in locations containing nanodroplets while no cavitation was observed in regions without nanodroplets. Ultrasound imaging (bottom) demonstrated nanodroplet guided cavitation treatment can be monitored in real-time with bubbles appearing as dynamically changing hyperechoic regions.

\section{Bubble Behavior}

The bubble expansion and collapse observed in histotripsy is substantially more energetic than traditionally defined inertial cavitation where the maximal bubble diameter becomes equal or greater than twice the initial bubble size [53]. In histotripsy, microbubbles formed from preexisting nuclei $<10 \mathrm{~nm}$ grow to over $50 \mu \mathrm{m}$ before violently collapsing, all occurring within $100 \mu \mathrm{s}$. This bubble behavior is critical to achieve cell disruption. To study the behavior of cavitation bubbles generated by nanodroplet-mediated histotripsy, optical images of bubbles were recorded by the high-speed camera at different time delays after the arrival of the histotripsy pulse. The peak 
negative pressure of $15.6 \mathrm{MPa}$ was used, because it is the lowest pressure at which a cavitation cloud was always generated $\left(\mathrm{P}_{\mathrm{cav}}=1\right)$ using nanodroplets. In agarose gel, the bubble diameter increased to the maximum diameter of $311.2 \pm 49.9 \mu \mathrm{m}$ at $21 \mu$ s (Figure 8 ). The average bubble lifespan from the arrival of the histotripsy pulse to when the bubble became too small to be observed on the optical image was measured to be $\sim 60 \mu$ s (Figure 7). This level of bubble expansion and collapse is similar to the behavior of bubbles formed using histotripsy alone at higher pressures and is anticipated to create cell disruption.

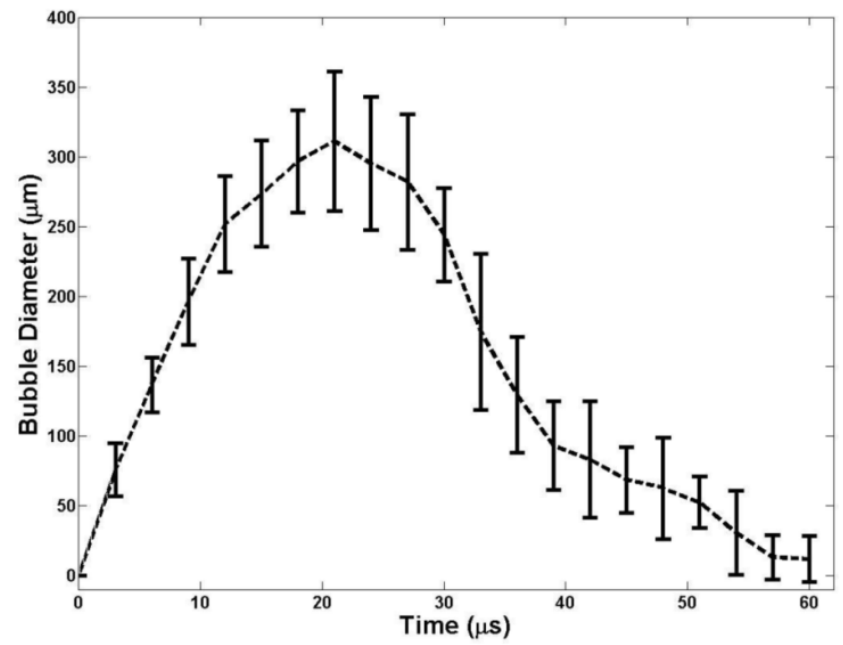

Fig 8. A plot of bubble behavior in agarose tissue phantoms. The diameter of bubble generated using a 2 -cycle $500 \mathrm{kHz}$ pulse at I5MPa as a function of time. At each time point, the average bubble diameter and standard deviation are plotted.

\section{Ablation of Red Blood Cells in Tissue Phantom}

Agarose tissue phantoms embedded with RBC layers were used to evaluate the ability of nanodroplet-mediated histotripsy to create cell fractionation, as lesions visualized in RBC phantoms have been shown to be similar to those generated in tissue identified by histology [44]. Nanodroplet-mediated histotripsy resulted in consistent, well-defined lesions in RBC phantoms, similar to those observed for histotripsy therapy, but at pressure levels well below the histotripsy intrinsic threshold. At peak negative pressure below $10 \mathrm{MPa}$, no microbubbles were observed and no lesions were visualized. At peak negative pressure of $11.0 \mathrm{MPa}$ and greater, when cavitation bubbles were generated, well-defined lesions were formed in the RBC phantoms using nanodroplets combined with histotripsy (Figure 9). Nanodroplet-mediated histotripsy fractionated the RBC phantom with similar efficiency to histotripsy at higher pressure with all RBCs within the focal volume completely fractionated within 2,000 pulses, resulting in transparent lesions with no intact RBCs remaining. The lesion area was analyzed following the procedure described in the Experimental Section demonstrating the area of lesion in the RBC layer increased with increasing pressure. For example, the lesion areas for RBC phantoms containing nanodroplets $(n=6)$ were $0.77 \pm 0.09 \mathrm{~mm}^{2}, 3.92 \pm 0.41 \mathrm{~mm}^{2}$, and $6.27 \pm 0.86 \mathrm{~mm}^{2}$ for peak negative pressures of $11.0 \mathrm{MPa}, 15.9 \mathrm{MPa}$, and 20.7 MPa, respectively (Figure 9). Furthermore, lesion area was plotted as a function of pulse number demonstrating $>75 \%$ of the focal region was fractionated within the first 1,000 pulses and $>95 \%$ of the focal region fractioned after 1,500 pulses at all pressure levels treated (Figure 10). In comparison, in control gels without nanodroplets, no lesions were formed at any treatments in this pressure range. These results are important to show that nanodroplet-mediated cavitation can indeed create cell disruption with the same effectiveness (i.e., the same number of pulses) as using histotripsy alone but at a significantly lower pressure. This is an important validation of our hypothesis confirming that the cavitating microbubbles generated via nanodroplets are destructive to use for ablation.

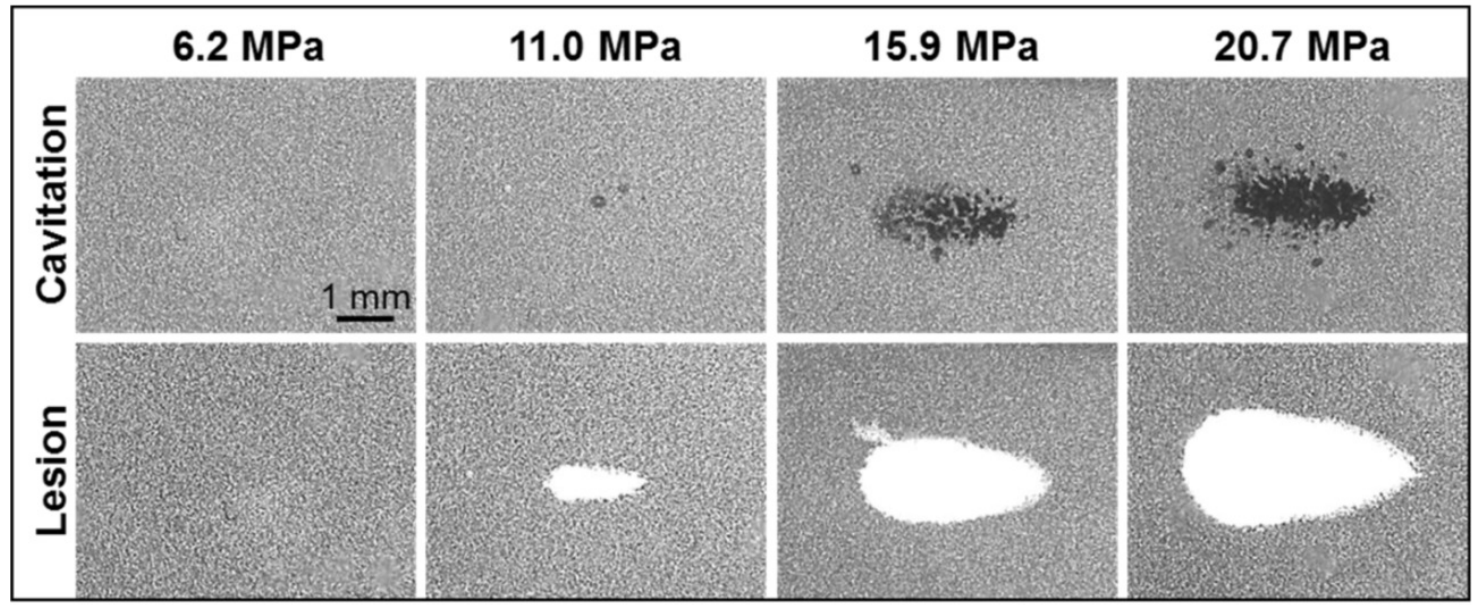

Fig 9. Optical images of nanodroplet-mediated cell fractionation. Images of cavitation bubble cloud (dark) and lesions (white) generated in the RBC agarose gel (grey) using nanodroplet-mediated histotripsy at different pressure levels. A total of 2000 2-cycle pulses at $10 \mathrm{~Hz}$ PRF were used for each treatment. 


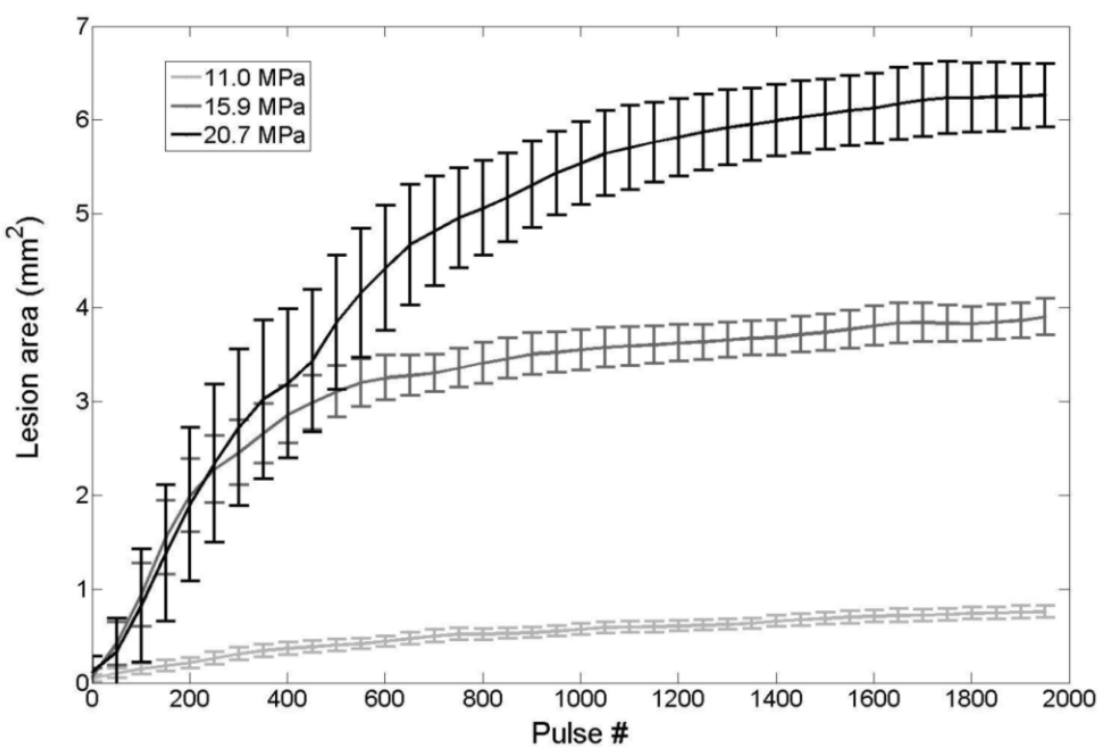

Fig 10. Nanodroplet-mediated lesion formation. The lesion area formed in the RBC agarose gel as a function of pulse number for treatments at II.0, I5.9, and 20.7 MPa. No damage was formed for treatments at 6.2 MPa.

The findings in this work demonstrated proof of concept for selective cavitational ablation by combining histotripsy with nanodroplets. Our nanodroplets are in the size range required to penetrate through the leaky tumor vasculature and have been shown to decrease the pressure threshold required for histotripsy. These initial experiments demonstrate the potential of selectively applying histotripsy to cancer cells by using an appropriate pressure level. Furthermore, by using a low frequency transducer with a large focal zone, it is possible to apply histotripsy simultaneously to a large focal volume with cavitation occurring only at locations containing nanodroplets. As such, the treatment time for a larger volume of tissue would be shortened while still maintaining high treatment selectivity to cancer cells through the targeted nanodroplets.

\section{Conclusions}

In this work, the feasibility of using PFP encapsulated nanodroplets for enhanced histotripsy ablation was investigated. Results support our hypothesis that nanodroplets significantly decrease the histotripsy threshold to form a cavitation bubble cloud while maintaining the effectiveness of histotripsy tissue ablation. Nanodroplet-mediated histotripsy was shown capable of creating microbubble expansion and collapse as well as well-defined ablation similar to histotripsy but at significantly lower pressure. To build upon this initial study, future work will aim to test the hypothesis that our nanodroplets can be functionalized to bind to cancer cells in vitro and demonstrate that nanodroplet-mediated histotripsy can selectively fractionate cancer cells. We will also investigate that using nanodroplets delivered to the capsule of tumor nodules, the tumor nodule can be fractionated from outside to the inner core. Furthermore, future experiments will aim to show that nanodroplets can be delivered and accumulated at a tumor site for the selective histotripsy ablation of cancer cells in a relevant in vivo cancer model.

\section{Acknowledgements}

Eli Vlaisavljevich was supported by a National Science Foundation Graduate Research Fellowship. This work was supported by a grant from the United States Department of Defense (W81XWH-11-PCRPID).

\section{Competing Interests}

The authors have declared that no competing interest exists.

\section{References}

1. Campbell RB. Tumor physiology and delivery of nanopharmaceuticals. Anticancer Agents Med Chem. 2006; 6: 503-12.

2. Hobbs SK, Monsky WL, Yuan F, Roberts WG, Griffith L, Torchilin VP, et al. Regulation of transport pathways in tumor vessels: role of tumor type and microenvironment. Proc Natl Acad Sci U S A. 1998; 95: 4607-12.

3. Torchilin V. Tumor delivery of macromolecular drugs based on the EPR effect. Adv Drug Deliv Rev. 2011; 63: 131-5.

4. Fang J, Nakamura H, Maeda H. The EPR effect: Unique features of tumor blood vessels for drug delivery, factors involved, and limitations and augmentation of the effect. Adv Drug Deliv Rev. 2011; 63: 136-51.

5. Gao Z, Kennedy AM, Christensen DA, Rapoport NY. Drug-loaded nano/microbubbles for combining ultrasonography and targeted chemotherapy. Ultrasonics. 2008; 48: 260-70. Epub 2007 Nov 19.

6. Kawabata Ki, Asami R, Yoshikawa H, Azuma T, Umemura Si. Sustaining Microbubbles Derived from Phase Change Nanodroplet by Low-Amplitude Ultrasound Exposure. Jpn J Appl Phys. 2010; 49: 07HF20.

7. Sheeran PS, Luois S, Dayton PA, Matsunaga TO. Formulation and acoustic studies of a new phase-shift agent for diagnostic and therapeutic ultrasound. Langmuir. 2011; 27: 10412-20.

8. Sheeran PS, Wong VP, Luois S, McFarland RJ, Ross WD, Feingold S, et al. Decafluorobutane as a Phase-Change Contrast Agent for Low-Energy Extravascular Ultrasonic Imaging. Ultrasound Med Biol. 2011; 37: 1518-30. 
9. Du L, Jin $\mathrm{Y}$, Zhou W, Zhao J. Ultrasound-Triggered Drug Release and Enhanced Anticancer Effect of Doxorubicin-Loaded Poly(D,L-Lactide-co-Glycolide)-Methoxy-Poly(Ethylene Glycol) Nanodroplets. Ultrasound Med Biol. 2011; 37: 1252-58.

10. Rapoport N, Nam KH, Gupta R, Gao Z, Mohan P, Payne A, et al. Ultrasound-mediated tumor imaging and nanotherapy using drug loaded, block copolymer stabilized perfluorocarbon nanoemulsions. J Control Release. 2011; 153: 4-15.

11. Rapoport NY, Nam KH, Gao Z, Kennedy A. Application of Ultrasound for Targeted Nanotherapy of Malignant Tumors. Acoust Phys. 2009; 55: 594-601.

12. Gao Z, Kennedy AM, Christensen DA, Rapoport NY. Drug-loaded nano/microbubbles for combining ultrasonography and targeted chemotherapy. Ultrasonics. 2008; 48: 260-70.

13. Kripfgans OD, Fowlkes JB, Miller DL, Eldevik OP, Carson PL. Acoustic droplet vaporization for therapeutic and diagnostic applications. Ultrasound Med Biol. 2000; 26: 1177-89.

14. Sheeran PS, Wong VP, Luois S, McFarland RJ, Ross WD, Feingold S, et al. Decafluorobutane as a phase-change contrast agent for low-energy extravascular ultrasonic imaging. Ultrasound Med Biol. 2011; 37: 1518-30.

15. Kawabata K, Asami R, Axuma T, Umemura S. Acoustic response of microbubbles derived from phase-change nanodroplet. Jpn J Appl Phys. 2010; 49: 07HF18.

16. Sheeran PS, Luois S, Dayton PA, Matsunaga TO. Formulation and acoustic studies of a new phase-shift agent for diagnostic and therapeutic ultrasound. Langmuir. 2011; 27: 10412-20.

17. Wilson K, Homan K, Emelianov S. Biomedical photoacoustics beyond thermal expansion using triggered nanodroplet vaporization for contrast-enhanced imaging. Nat Commun. 2012; 3: 618

18. Kawabata Ki, Asami R, Azuma T, Yoshikawa H, Umemura Si. Cavitation assisted HIFU with phase-change nano droplet. IEEE International Ultrasonics Symposium. 2008;: 780-3.

19. Kopechek JA, Zhang P, Burgess MT, Porter TM. Synthesis of phase-shift nanoemulsions with narrow size distributions for acoustic droplet vaporization and bubble-enhanced ultrasound-mediated ablation. J Vis Exp. 2012;: e4308.

20. Zhang M, Fabiilli ML, Haworth KJ, Padilla F, Swanson SD, Kripfgans OD, et al. Acoustic droplet vaporization for enhancement of thermal ablation by high intensity focused ultrasound. Acad Radiol. 2011; 18: 1123-32.

21. Kieran K, Hall TL, Parsons JE, Wolf JS, Jr., Fowlkes JB, Cain CA, et al. Refining histotripsy: defining the parameter space for the creation of nonthermal lesions with high intensity, pulsed focused ultrasound of the in vitro kidney. J Urol. 2007; 178: 672-6.

22. Winterroth F, Xu Z, Wang TY, Wilkinson JE, Fowlkes JB, Roberts WW, et al. Examining and analyzing subcellular morphology of renal tissue treated by histotripsy. Ultrasound Med Biol. 2011; 37: 78-86.

23. Xu Z, Fan Z, Hall TL, Winterroth F, Fowlkes JB, Cain CA. Size measurement of tissue debris particles generated from pulsed ultrasound cavitational therapy-histotripsy. Ultrasound Med Biol. 2009; 35: 245-55.

24. $\mathrm{Xu} \mathrm{Z}$, Fowlkes JB, Cain CA. A new strategy to enhance cavitational tissue erosion using a high-intensity, Initiating sequence. IEEE Trans Ultrason Ferroelectr Freq Control. 2006; 53: 1412-24.

25. Xu Z, Fowlkes JB, Rothman ED, Levin AM, Cain CA. Controlled ultrasound tissue erosion: the role of dynamic interaction between insonation and microbubble activity. J Acoust Soc Am. 2005; 117: 424-35.

26. Xu Z, Ludomirsky A, Eun LY, Hall TL, Tran BC, Fowlkes JB, et al. Controlled ultrasound tissue erosion. IEEE Trans Ultrason Ferroelectr Freq Control. 2004; 51: 726-36.

27. Maxwell AD, Owens G, Gurm HS, Ives K, Myers DD, Jr., Xu Z. Noninvasive treatment of deep venous thrombosis using pulsed ultrasound cavitation therapy (histotripsy) in a porcine model. J Vasc Interv Radiol. 2011; 22: 369-77.

28. Xu Z, Owens G, Gordon D, Cain C, Ludomirsky A. Noninvasive creation of an atrial septal defect by histotripsy in a canine model. Circulation. 2010; 121: 742-9.

29. Durmaz H, Dag A, Altintas O, Erdogan T, Hizal G, Tunca U. One-pot synthesis of $\mathrm{ABC}$ type triblock copolymers via in situ click $3+2$ and Diels-Alder 4+2 reactions. Macromolecules 2007; 40: 191-98.

30. Durmaz YY, Vlaisavljevich E, Xu Z, Elsayed MEH. Development of targeted nano-bubbles for ultrasound imaging and ablation of prostate cancer. $35^{\text {th }}$ Annual Symposium of Macromolecular Science \& Engineering. Ann Arbor, Michigan. 2011

31. Durmaz YY, Vlaisavljevich E, Xu Z, ElSayed MEH. Development of nanodroplets for targeted ultrasound-mediated ablation of cancer. Engineering Graduate Symposium. Ann Arbor, Michigan. 2011.

32. Bartczak D, Kanaras AG. Preparation of peptide-functionalized gold nanoparticles using one pot EDC/sulfo-NHS coupling. Langmuir. 2011; 27: 10119-23.

33. Nam K, Kimura T, Kishida A. Controlling coupling reaction of EDC and NHS for preparation of collagen gels using ethanol/water co-solvents. Macromol Biosci. 2008; 8: 32-7.

34. Wildling L, Unterauer B, Zhu R, Rupprecht A, Haselgrubler T, Rankl C, et al. Linking of sensor molecules with amino groups to amino-functionalized AFM tips. Bioconjug Chem. 2011; 22: 1239-48.

35. Yang X, Church CC. A model for the dynamics of gas bubbles in soft tissue. J Acoust Soc Am. 2005; 118: 3595-606.
36. Maxwell AD, Cain CA, Hall TL, Fowlkes JB, Xu Z. Probability of cavitation for single ultrasound pulses applied to tissues and tissue-mimicking materials. Ultrasound Med Biol. 2013; 39: 449-65.

37. Sheeran PS, Dayton PA. Phase-change contrast agents for imaging and therapy. Curr Pharm Des. 2012; 18: 2152-65.

38. Masuzaki R, Tateishi R, Yoshida H, Sato T, Ohki T, Goto T, et al. Assessing liver tumor stiffness by transient elastography. Hepatol Int. 2007; 1: 394-7.

39. Zhang M, Nigwekar P, Castaneda B, Hoyt K, Joseph JV, di Sant'Agnese A, et al. Quantitative characterization of viscoelastic properties of human prostate correlated with histology. Ultrasound Med Biol. 2008; 34: 1033-42.

40. Friedman M, Strauss M, Amendt P, London R, Glinsky M. Two-dimensional Rayleigh model for bubble evolution in soft tissue. Phys Fluids. 2002; 14: 1768-80.

41. Ayme EJ, Carstensen EL. Cavitation induced by asymmetric distorted pulses of ultrasound: theoretical predictions. IEEE Trans Ultrason Ferroelectr Freq Control. 1989; 36: 32-40.

42. Parsons JE, Cain CA, Fowlkes JB. Cost-effective assembly of a basic fiber-optic hydrophone for measurement of high-amplitude therapeutic ultrasound fields. J Acoust Soc Am. 2006; 119: 1432-40.

43. Normand V, Lootens DL, Amici E, Plucknett KP, Aymard P. New insight into agarose gel mechanical properties. Biomacromolecules. 2000; 1: 730-8.

44. Maxwell AD, Wang TY, Yuan L, Duryea AP, Xu Z, Cain CA. A tissue phantom for visualization and measurement of ultrasound-induced cavitation damage. Ultrasound Med Biol. 2010; 36: 2132-43.

45. O'Reilly RK. Spherical polymer micelles: nanosized reaction vessels? Phil. Trans. R. Soc. A. 2007; 365: 2863-78.

46. Zhulina EB, Adam M, LaRue I, Sheiko SS, Rubinstein M. Diblock Copolymer Micelles in a Dilute Solution. Macromolecules. 2005; 38: 5330-51.

47. Matyjaszewski K, Xia J. Atom Transfer Radical Polymerization. Chem Rev. 2001; 101: 2921-90.

48. Gao Z, Kennedy AM, Christensen DA, Rapoport NY. Drug-loaded nano/microbubbles for combining ultrasonography and targeted chemotherapy. Ultrasonics. 2008; 48: 260-70.

49. Shiraishi K, Endoh R, Furuhata H, Nishihara M, Suzuki R, Maruyama K, et al. A facile preparation method of a PFC-containing nano-sized emulsion for theranostics of solid tumors. Int J Pharm. 2011; 421: 379-87.

50. Wang C-H, Kang S-T, Lee Y-H, Luo Y-L, Huang Y-F, Yeh C-K. Aptamer-conjugated and drug-loaded acoustic droplets for ultrasound theranosis. Biomaterials. 2012; 33: 1939-47.

51. Apfel RE, Holland CK. Gauging the likelihood of cavitation from short-pulse, low-duty cycle diagnostic ultrasound. Ultrasound Med Biol. 1991; 17: 179-85.

52. Carstensen EL, Gracewski S, Dalecki D. The search for cavitation in vivo. Ultrasound Med Biol. 2000; 26: 1377-85.

53. Leighton TG. The Acoustic Bubble. Academic Press. 1994 